\title{
An operant arena for rats
}

\author{
STEPHEN R. GOLDSTEIN \\ Lakehead University, Thunder Bay, Ontario P7B SEI, Canada
}

A multiunit operant arena for the study of dispersion patterns in rats is described. Lawful relationships between the spatiotemporal organization of the group and reinforcement schedules have been found. The scientific and humanistic advantages of the system are discussed.

The distribution and abundance of animals is so central a feature of social organization (Krebs, 1978) that its analysis forms the core of ecological science. Ecological analysis lays considerable emphasis on the role of food supply in regulating population density patterns and yet the mechanisms by which this is achieved still require clarification (Watson \& Moss, 1970). It is therefore noteworthy that when animals go about the business of procuring food, they do so with respect to a schedule of reinforcement fabricated by their natural and social environment. Important features of such schedules can be simulated in an operant arena, and their effects on dispersion patterns can be analyzed.

\section{THE OPERANT ARENA}

A $3.7 \times 3.4 \times 1.2 \mathrm{~m}$ arena constitutes the permanent living and work area for a group of eight male hooded rats (Figure 1). Hinged Plexiglas partitions along the two opposite walls of the arena permit observation from four cubicles and access to the arena. Mirrors, $1.23 \times .31 \mathrm{~m}$, mounted on the walls above these partitions permit observation of the entire arena from any cubicle. Ventilation is achieved by a large exhaust fan situated over a drop pegboard ceiling of the arena. A suspended $1.22-\mathrm{cm}$ wire-mesh floor facilitates the flow of air through the system and also permits urine and feces to fall onto a V-shaped trough. Perforated copper pipes (1.9-cm diam) along the top of the urinal discharge water periodically through the night when a solenoid control valve is pulsed by a timer.

Two banks of fluorescent lights, mounted in the pegboard ceiling, provide illumination for a $14-\mathrm{h} / 10 \mathrm{~h}$ light-dark cycle. A pair of elevated wire rest shelves $(55 \times 35 \times 36 \mathrm{~cm})$ fitted with an access ladder are situated on opposite sides of the arena for the animal's convenience and comfort.

Eight feeding stations are arranged along two opposite ends of the arena in a bilateral symmetric pattern. A feeding station consists of an insertable response bar

I thank Gail Zawette of Lakehead University Media Services for her drawing of the rat arena. This research was supported in part by National Science and Engineering Research Council of Canada (NSERC) Grant A7451 and by the President's NSERC Fund.

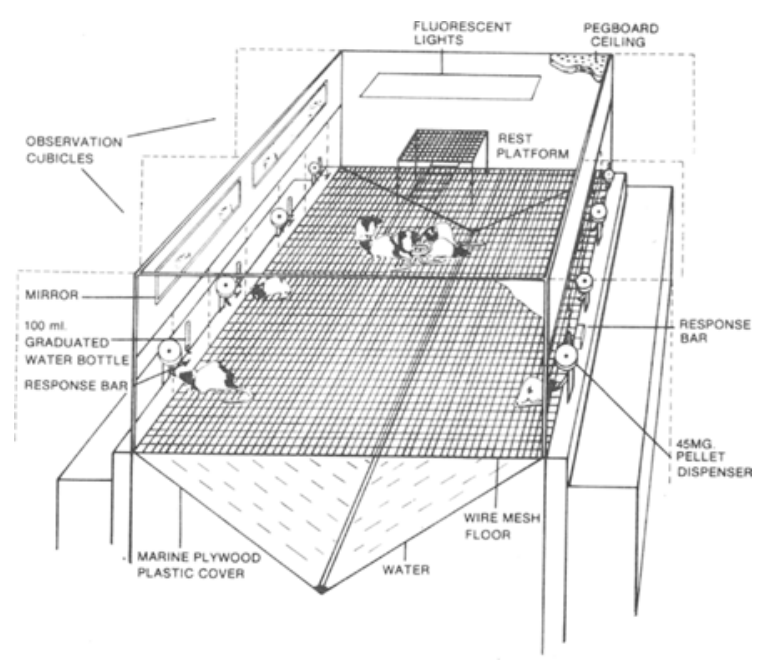

Figure 1. Schematic representation of eight-rat eight-station operant arena.

(Gerbrands Model GG 312), a 45-mg pellet dispenser (Gerbrands Model D-1), a food cup, and a 100-ml graduated water bottle. A Pet 2001 series microprocessor provides station-by-station control of reinforcement schedules, data acquisition, and data analysis (Blekkenhorst, Mayes, \& Goldstein, Note 1).

\section{EXPERIMENTAL PROCEDURES}

Animals are typically exposed to two daily $30-\mathrm{min}$ feeding sessions at 10:30 a.m. and 4:30 p.m. Sessions commence with the manual insertion of the levers through a small opening in the Plexiglas partitions and end with the levers being withdrawn and the openings covered with a Plexiglas baffle. Barpresses result in pellet delivery according to the schedule of reinforcement programmed for a station. At the beginning of each minute, a visual scan of the eight stations is made by an observer and the number of rats observed at each station is recorded on a dispersion data chart (DDC). The natural marking patterns of the rats' backs permit individual identification, but this type of data can be accurately recorded only under certain schedule conditions. 


\section{DENSITY, DISPERSION, AND STABILITY}

A computer analysis (Blekkenhorst et al., Note 1) of the DDC generates information about density, dispersion, and stability patterns, as follows.

\section{Density}

Two measures of station density are obtained. One, the mean number of rats at a station (i.e., N. Station $1 /$ 30), gives an absolute measure of density. The other, the probability of rats per station (i.e., N. Station $1 /$ $\mathrm{N}$. Station ${ }_{1} \ldots 8$ ), provides a measure of density relative to all stations in the system.

\section{Dispersion}

When all rats are simultaneously working at separate stations, they form the most regular dispersion (Poole, 1974) pattern possible. At the other extreme, when all eight rats simultaneously work at one station, they form the most clumped or contagious (Poole, 1974) pattern possible. Between these extremes, there are 20 other dispersion possibilities for the eight-rat situation if station and rat identities are ignored. For conditions in which fewer than eight rats work, 44 possible patterns exist. The probabilities associated with the combined total of 66 patterns can be obtained from a computer analysis of the DDC.

\section{Stability Analysis}

If information about station identity is preserved, at least four measures of the stability of the dispersion patterns can be obtained. An identical transition, which is regarded as the most conservative estimate of stability, specifies a condition in which the number of rats colonizing specific stations does not change from one minute to the next, for example, (1) 0-0-4-0-2-1-0-1, (2) 0-0-4-02-1-0-1. A somewhat less conservative estimate of stability is the position transition, in which the density pattern remains the same, but the location of the pattern changes, for example, (1) $0-0-4-0-2-1-0-1$, (2) 4-2-0-1-1$0-0-0$. Measures of increasingly more volatile change are the microstate and macrostate transitions. The microstate transition specifies a change in which a specific number of rats colonize a specific number of stations in different configurations from one minute to the next, for example, (1) $0-0-4-2-1-1-0-0$, (2) 0-0-2-2-2-2-0-0. The macrostate transition specifies a change in which a specific number of rats colonize a different number of stations in 2 consecutive minutes, for example, (1) $0-0-0-4-2-0-1-1$, (2) $0-0-5-2-0-1-0-0$. The probabilities and conditional probabilities of these and other types of changes are all readily obtained from computer analysis of the DDC.

\section{EFFECTS OF SCHEDULES}

In one study (Goldstein \& Mazurski, Note 2), an ascending series of FR values (FR 1, FR 4, FR 8, FR 16, and FR 32) was presented to the group to determine
Table 1

Frequency of Dispersion Patterns for an FR 4 Schedule

\begin{tabular}{|c|c|c|c|c|}
\hline \multirow[b]{2}{*}{ Category } & \multicolumn{2}{|c|}{ This Session } & \multicolumn{2}{|c|}{ Cumulative Sessions } \\
\hline & Total & Percent & Total & Percent \\
\hline 8 & 0 & 0 & 0 & 0 \\
\hline 17 & 0 & 0 & 0 & 0 \\
\hline 26 & 0 & 0 & 0 & 0 \\
\hline 35 & 0 & 0 & 0 & 0 \\
\hline 44 & 0 & 0 & 0 & 0 \\
\hline 116 & 0 & 0 & 0 & 0 \\
\hline 125 & 0 & 0 & 0 & 0 \\
\hline 134 & 0 & 0 & 1 & 0 \\
\hline 224 & 0 & 0 & 0 & 0 \\
\hline 233 & 0 & 0 & 0 & 0 \\
\hline 2222 & 0 & 0 & 1 & 0 \\
\hline 1115 & 0 & 0 & 0 & 0 \\
\hline 1124 & 0 & 0 & 0 & 0 \\
\hline 1133 & 0 & 0 & 0 & 0 \\
\hline 1223 & 0 & 0 & 1 & 0 \\
\hline 11114 & 0 & 0 & 9 & 1 \\
\hline 11123 & 2 & 8 & 53 & 9 \\
\hline 11222 & 2 & 8 & 27 & 4 \\
\hline 111113 & 1 & 4 & 40 & 6 \\
\hline 111122 & 13 & 54 & 176 & 30 \\
\hline 1111112 & 6 & 25 & 264 & 45 \\
\hline 11111111 & 0 & 0 & 14 & 2 \\
\hline Odd Dispersions & 6 & 20 & 74 & 11 \\
\hline
\end{tabular}

how increased work requirements affect dispersion. Sample data of the percent frequency and cumulative percent frequency for the 22 dispersion patterns are shown for 1 day of the FR 4 series in Table 1. Although the rats were free to work under the most optimal condition, or the most regular dispersion, they adopted this mode only $2 \%$ of the total time, preferring varying states of aggregation. Under low-ratio conditions, clumping is maintained because ratio schedules maintain response rates high enough to feed small aggregates (Baron \& Littman, 1961; Mowrer, 1940).

Ascending interval schedules that concurrently pay off at the same values (Goldstein, Johnson, \& Ward, Note 3 ) and ratio schedules that concurrently pay off at different values (Goldstein, Note 4) have also been investigated. A consistent finding is that the matching law (Herrnstein, 1970) applies not only to group responding (Baum, 1974; Graft, Lea, \& Whitworth, 1977), but also to the density of animal groupings.

Dispersion patterns, which normally exhibit a high degree of species specificity (Odum, 1963), have been found to be pliable in the arena. Thus regular, random, or clumped dispersions can be imposed on the same group of subjects by the appropriate schedule of reinforcement. Similarly, the vital business of procuring food can be done cooperatively, competitively, or independently of conspecifics. It is likewise possible to generate all of these options in the same group of animals in the arena. Apparently, the species-typical nature of food procurement and dispersion strategies may be due to more or less stable features of the speciestypical environment, in this case to the contingencies of reinforcement engendered by the acquisition of food. 


\section{DISCUSSION}

Perhaps more important than any particular set of findings are the advantages that the operant arena offers a science of social behavior. It complements the approach of bringing the operant laboratory into the animals' natural environment (Baum, 1974; Smith, 1965). In addition to expanding the domain of traditional operant analysis, it provides a common base for interdisciplinary research involving ethology, sociobiology, and economics. Since the rats are free to search for food in clearly defined areas and to work for food under known conditions of reinforcement, cost-benefit theories, such as optimal foraging strategies (Schoener, 1971), can be readily tested. Continued refinements in the arena, by the addition of females, the addition of qualitatively different reinforcers, and the use of telemetry systems, will help clarify a number of issues and create the possibility for challenging experiments in social dynamics.

The self-contained self-sustaining system also has advantages for the animals on both sides of the Plexiglas partitions. Inside, the animals do not have to be confined in small cages, isolated from conspecifics, handled by nervous experimenters, food deprived to work, or "sacrificed" at the end of an experiment. On the contrary, the full range of social behavior may be conveniently studied while those animals on the outside are exposed to a fascinating view of animal behavior.

\section{REFERENCE NOTES}

1. Blekkenhorst, H. J., Mayes, L., \& Goldstein, S. R. Computerization of the operant arena. Manuscript in preparation, 1981.
2. Goldstein, S. R., \& Mazurski, E. Fixed-ratio control and dispersion. Manuscript in preparation, 1981.

3. Goldstein, S. R., Johnson, P. A., \& Ward, G. A new look at an old problem: Schedule control of dispersion. Manuscript in preparation, 1981.

4. Goldstein, S. R. Schedule control of dispersion patterns. Paper presented at the meeting of the Animal Behavior Society, Seattle, Wash., 1978.

\section{REFERENCES}

Baron, A., \& Littman, R. Studies of individual and paired interaction problem-solving behavior of rats: II. Solitary and social controls. Genetic Psychology Monographs, 1961, 64, 129-209.

Baum, W. M. Choice in free ranging wild pigeons. Science, 1974, 185, 78-79.

Graft, D. A., Lea, S. E. G., \& Whitworth, T. L. The matching law in and within groups of rats. Journal of the Experimental Analysis of Behavior, 1977, 25, 183-184.

Herrnstein, R. J. On the law of effect. Journal of the Experimental Analysis of Behavior, 1970, 13, 243-266.

KrEBS, C. J. Ecology: The experimental analysis of distribution and abundance. New York: Harper \& Row, 1978.

Mowner, O. H. Animal studies in the genesis of personality. Transactions of the New York Academy of Science, 1940, 3, 8-11.

Odum, E. Ecology. New York: Holt, Rinehart, \& Winston, 1963.

Poole, R. W. An introduction to quantitative ecology. New York: McGraw-Hill, 1974.

Schoener, T. W. Theory of feeding strategies. Annual Review of Ecology and Systematics, 1971, 2, 369-404.

Smith, R. F. Laboratory control of behavior without laboratory restraint. Psychonomic Science, 1965, 3, 537-538.

Watson, A., \& Moss, R. Dominance, spacing behavior and aggression in relation to population limitation in vertebrates. In A. Watson (Ed.), Animal populations in relation to their food resources. Oxford: Blackwell, 1970.

(Accepted for publication March 2, 1981.) 